\title{
Effects of Humidity Exposure on a Solid Dosage Form Dissolution Performance.
}

\author{
Craig A. J. Kemp* and Michael J Skibic* \\ *Eli Lilly and Company, Lilly Corporate Center, Indianapolis, Indiana 46285, USA
}

Suppression in the extent of release of active pharmaceutical ingredient (API) from tablets during dissolution is observed after exposure to high humidity. The API salt can exist as several polymorphs. The one used for the tablet manufacture ('Form C') is known to under go hydration upon exposure to conditions of elevated humidity and temperature. Two hydrates are known to form and these are designated as Forms A or B. Form A is known to be more thermodynamically stable at moderate humidity levels whereas Form B is most stable at elevated humidity levels. The dissolution profiles of tablets prepared using Forms A and B are not significantly different from Form $\mathrm{C}$ under the test conditions, so the differences observed in the tablets could not be directly explained by hydration alone. While the actual disintegration times were largely unaffected, it was found that during dissolution, disintegration of moisture exposed tablets resulted in larger drug product particles. Form B tablets exposed to elevated humidity levels do not exhibit suppressed dissolution. Several microanalytical techniques were employed to further investigate the effects of moisture exposure on the tablets.

Xray micro CT images were obtained of intact tablets that had been exposed to different humidity levels (Figure 1). The tablet stored under desiccating conditions exhibits a few cracks through the interior. The tablet stored under high humidity conditions appears to have more extensive interconnected cracks. A subtle difference in the appearance granularity of the interior can also be seen.

Tablets were embedded in an epoxy matrix then microtomed to expose the interior. These were analyzed by SEM and ToF-SIMS before being exposed to high humidity for a period of time that would normally guarantee the dissolution suppression effect. The cross-sectioned tablets were then reanalyzed.

The SEM micrographs (Figure 2) show that the humidity exposure resulted in the formation of "long needles". These are composed of API, as shown by EDS mapping of $\mathrm{Cl}$ and S, both of which, in this formulation, are unique to the drug substance. This phenomenon was not observed in tablets made with Form B API only when subjected to the same conditions.

ToF-SIMS data (Figure 3) suggest that, prior to moisture exposure; the polysorbate 80 is evenly distributed across the surface. After exposure to moisture, there is evidence that the polysorbate 80 pools. Because polysorbate 80 is added as a wetting agent for the drug substance, changes in the distribution of this excipient has the potential to impact dissolution.

Microanalytical techniques enabled the visualization of changes in tablet structure and component distribution, which could help explain moisture-induced dissolution changes although, phenomena occurring at a surface may not be representative of processes within the tablet core. 

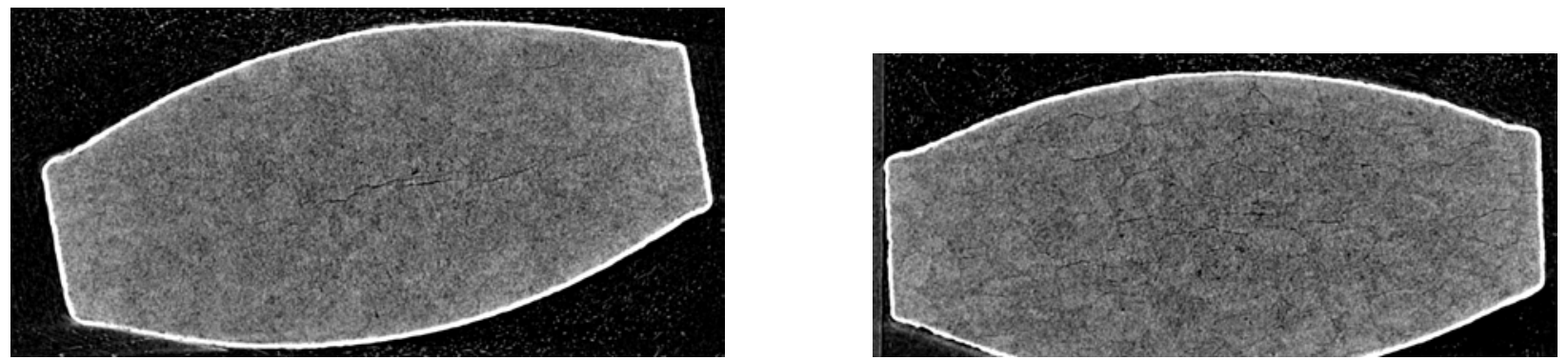

Figure 1. Xray micro CT reconstructed slices through whole tablets. Left: tablet kept under desiccating conditions. Right: tablet stored in $70 \% \mathrm{RH}$ conditions.

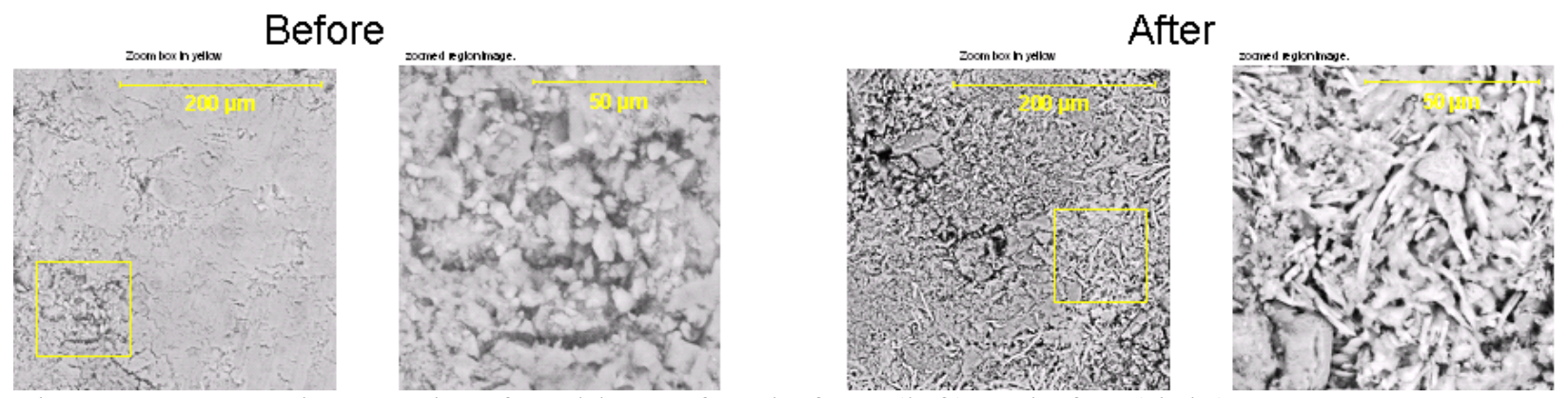

Figure 2. SEM micrographs of a tablet surface before (left) and after (right) exposure to $75 \%$ relative humidity at ambient lab conditions for about 1 week.

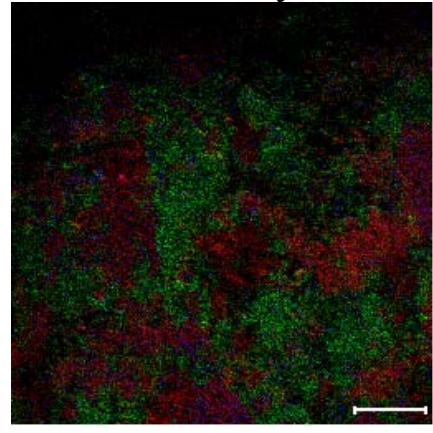

Scale 100um

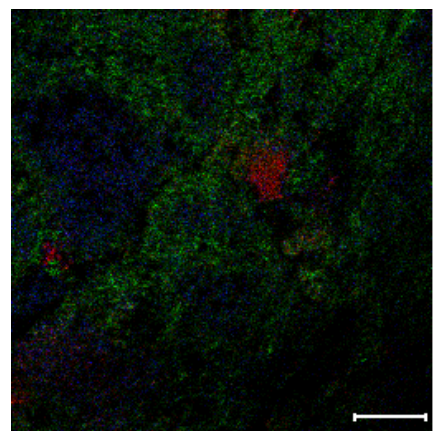

Scale 100 um

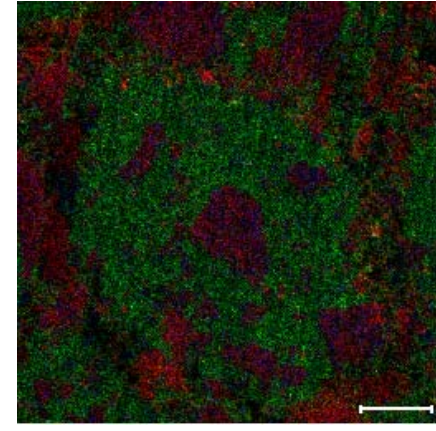

Scale 100um

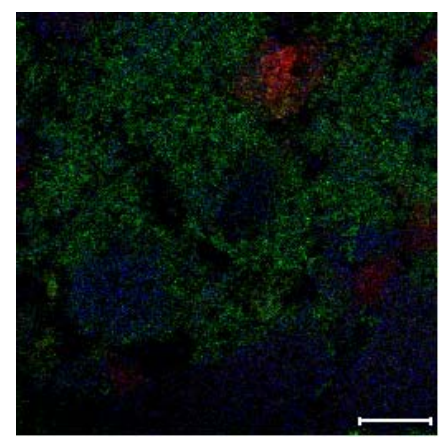

Scale 100 um

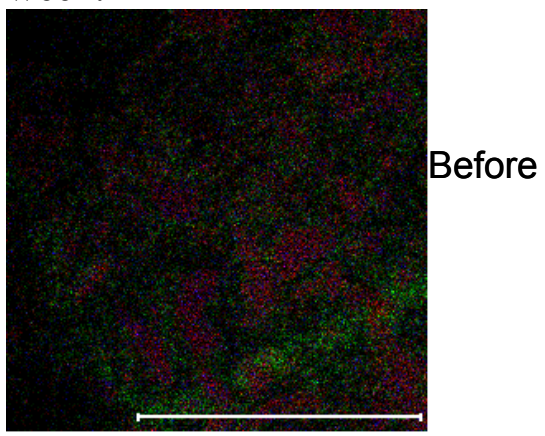

Scale $1 \mathrm{~mm}$

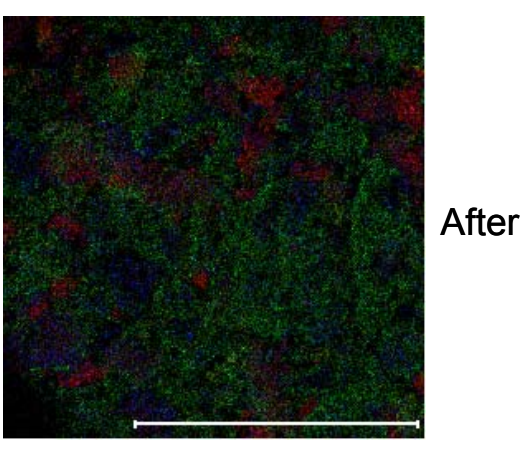

Scale $1 \mathrm{~mm}$

\section{R polysorbate GAPI B povidone}

Figure 3. TOF SIMS data - Upper three frames show the response before moisture exposure. The lower three plots show the distributions after moisture exposure. Key: Red $=$ polysorbate, Green $=$ API, Blue $=$ povidone 